\title{
The Latent Structure of Youth Responses to Peer Provocation
}

\author{
Melanie A. Dirks • Teresa A. Treat • V. Robin Weersing
}

Published online: 16 October 2010

(C) The Author(s) 2010. This article is published with open access at Springerlink.com

\begin{abstract}
This study examined whether the three categories often applied to children's behavior - aggressive, avoidant, and assertive - actually capture the structure of a naturalistic sample of youth behavior coded at a more micro level. A sample of lower-income youth $(N=392 ; M$ age $=12.69$, $S D=0.95$ ) completed a new multiple-choice measure asking them to select responses to scenarios depicting physical, verbal, and relational provocation by a peer. Youth responses to the vignettes showed the expected associations with self-reported aggression and regulation of anger, providing preliminary evidence for the convergent validity of the measure. Factor analysis confirmed that responses loaded on three factors: aggression, avoidance, and assertion. Model fit was adequate $(\mathrm{RMSEA}=.028)$ and crossvalidated in a second sample (RMSEA=.039). Several types of responses loaded on two factors suggesting that some strategies that youth use to manage provocation are not "pure" examples of these broadband categories. Implications for conceptualization and measurement of youth social behavior are discussed.
\end{abstract}

\footnotetext{
M. A. Dirks $(\bowtie)$

Department of Psychology,

McGill University,

1205 Dr. Penfield Avenue,

Montreal, Quebec H3A1B1, Canada

e-mail: melanie.dirks@mcgill.ca

\section{T. A. Treat}

University of Iowa,

Iowa City, IA, USA

V. R. Weersing

San Diego State University/University of California,

San Diego, CA, USA
}

Keywords Social skills · Peer provocation - Aggression . Assertion

\section{Introduction}

Increasingly, researchers are arguing for the utility of measuring youth behavior with respect to key social tasks (e.g., Rose and Asher 1999; McFall 1982). This approach is advantageous because the skills necessary for success may be quite situation specific; thus, children may do well in one interpersonal context but have difficulty in another (Dirks et al. 2007a). In order to improve children's social functioning, then, we might be better off focusing specifically on the skills required to manage important types of social situations, rather than using more global assessments of behavior.

When adopting a task-based approach to measurement, it is vital that we choose the right interpersonal contexts. Youth will confront an infinite number of social scenarios, but most will not yield interesting information about their social functioning. Goldfried and D'Zurilla (1969) posited that the most important tasks are those that are commonly occurring, difficult to manage, and critical (i.e., responding poorly will result in negative consequences). A significant body of work suggests that responding when provoked by a peer is one such task. Unfortunately, many children are victimized by peers: Between $30 \%$ and $60 \%$ of youth report being targeted aggressively within the last year at school (see Card and Hodges 2008), with harassment occurring at the hands of both classmates and friends (Crick and Nelson 2002). Responding effectively to provocation is difficult for many reasons; for example, being provoked is associated with strong affective responses, including anger and fear 
(e.g., Kochenderfer-Ladd 2004), which may make it harder to enact an effective response. Finally, children's responses to provocation have been shown to predict subsequent victimization, indicating that use of some strategies may serve to increase social difficulties (Kochenderfer and Ladd 1997).

Such data suggest that a measure assessing youth responses to provocation would be of potential value to both clinicians and researchers. Such an instrument could take a number of different forms. One strategy would involve presenting youth with vignettes describing provocation scenarios, and asking what they would say or do in those situations. Such studies have revealed that youth generate a wealth of different strategies in response to provocation, including physical aggression, verbal aggression, ending one's relationship with the aggressor, making a polite request, asking the person why they did it, and seeking adult intervention (e.g., Dirks et al. 2007b; Dodge et al. 2002; Hughes et al. 2004; Troop-Gordon and Asher 2005).

Another approach would be to ask youth to select or rate the strategies that they would use from a menu of possible responses (e.g., Elledge et al. 2010). When using this method it is important that the available responses represent the range of actual strategies generated by youth. If key behaviors are missing, results could be misleading, as youth may not be able to select the response(s) they would actually use. One method of determining the domain of youth responses is to use theoretically derived categories of youth social behavior. A number of vignette-based measures of youth social functioning have been developed using this methodology. Often, investigators use responses that reflect three broadband categories: aggressive/hostile, avoidant/withdrawn/submissive, and assertive/sociable (e.g., Deluty 1979; Rudolph et al. 1994).

Deluty (1979) argued that these categories capture a large percentage of possible responses to challenging interpersonal situations. Assertiveness is typically defined as "the ability to express one's thoughts and feelings, both positive and negative, in a non-hostile way and without violating the rights of others" (Ollendick 1983, p. 3). Examples of such behaviors include asking someone to stop doing something and requesting information (e.g., Ollendick 1983; Quiggle et al. 1992). Assertiveness is distinct from aggression, which is self-expression that is damaging or harmful to others (Deluty 1979). Examples of aggressive behaviors include physical aggression, verbal aggression, and relational aggression, in which an individual threatens another's social relationships (e.g., Tapper and Boulton 2004). Although assertive and aggressive behaviors are conceptually distinct, in practice the boundary between the two categories may not always be clear (see Ostrov et al. 2006). Assertive behaviors may contain "an implied threat of highly aversive behavior contingent on non-compliance" (Patterson et al. 1967, p. 4); for example, the statement "Don't do that again" could be considered assertive, but might also be heard as a threat of aggression. In contrast to assertive and aggressive behaviors, submissive or avoidant behaviors do not express a person's thoughts or feelings. Examples of such actions include failing to object to unreasonable behaviors and going to an adult rather than confronting the person directly (Deluty 1984).

Previous investigators, then, typically have adopted a conceptual approach to ensuring that the range of possible strategies is represented, as well as to selecting the specific responses. Typically, judges - such as experts, people in the environment (e.g., teachers or peers) or the researchers themselves - are provided with definitions of predetermined category structures (e.g., aggression) and asked to make decisions about which behaviors fit each grouping (e.g., Deluty 1979; Quiggle et al. 1992). One limitation of this approach is that responses that do not clearly belong in one category may be miscoded. For example, one strategy that youth generate in response to peer provocation is ending the relationship with the aggressor (Dirks et al. 2007b). Some authors view ending one's relationship with the aggressor as relationally aggressive (e.g., Delveaux and Daniels 2000). Such behavior, however, may also be a method of avoiding the problem. Miscategorizing such strategies will result in measurement error that will reduce the power of analyses designed to identify correlates of problematic behavior. Moreover, miscategorization may pose problems clinically, as it could contribute to a misunderstanding of the function of youth behavior. Researchers may also choose to discard behaviors that are not considered clear exemplars of a given category. Doing so may be advantageous if the goal is to create a measure of children's tendency towards a particular behavioral style. If, however, the goal is to examine how youth manage a particular social situation, eliminating behaviors actually used by youth will limit the utility of the measure.

As such, we adopted a different approach when developing the Peer Provocation Inventory-Multiple Choice (PPI-MC), a self-report measure of youth responses to peer provocation. Specifically, we paired eleven scenarios describing different types of peer provocation with strategies actually generated by youth. In previous work, youth reported what they would say or do in each situation (Dirks 2007; Dirks et al. 2007b). Eighty-percent of youth responses were coded reliably into eight descriptive categories: physical aggression, verbal aggression, ending relationship with aggressor, damaging aggressor's relationship with others, doing nothing, seeking an explanation, telling an adult, and stating that the provocation crossed personal limits. On the PPI-MC, each vignette is paired with one response from each 
descriptive category, as well as a response combining verbal aggression and seeking an explanation. This hybrid "hostilely assertive" response was included because our earlier work indicated a significant number of youth generated strategies of this type (e.g., "what's your problem?").

Our goal, then, was to include the full range of youth responses, an approach which should increase the external validity of the measure, and allow us to obtain the most veridical representation of what youth would actually do in these situations. As such, we did not attempt to cull the list of behaviors to those we thought were "pure" examples of each of the hypothesized broader categories of aggression, avoidance, and assertion. Our approach provided an opportunity to examine the latent structure that underlies youth responses. Rather than working "top down," from a set of definitions, we worked "bottom up" to determine whether youth endorsement of behaviors corresponded empirically to the assumed response categories of aggression, avoidance, and assertion. This analysis also allowed us to determine how specific behavioral strategies mapped onto these groupings, which may help provide empirical justification for the categorization of responses into one or more of these three categories.

\section{Goals of Current Study}

In the current study, we aimed to address three goals. First, we determined whether the three-factor structure so often applied to youth behavior - aggressive, avoidant, and assertiveactually captured the structure of youth responses coded into more micro, descriptive categories. Previous studies have validated these categories only indirectly, by examining individual differences in endorsement of behaviors identified as aggressive, avoidant, and assertive (e.g., Quiggle et al. 1992; Rudolph et al. 1994). Examination of the structure of actual strategies generated by youth extends such work by evaluating this putative categorization explicitly. Second, we examined how youth responses to peer provocation mapped onto these latent constructs. Third, we obtained preliminary evidence for the convergent validity of the PPI-MC.

Factor-analytic techniques were used to assess our hypotheses about the factor structure and the loadings of specific behavioral strategies. We expected each strategy to load on at least one of three latent factors: aggression, avoidance, and assertion. For the aggression factor, we anticipated that physical aggression, verbal aggression, and damaging the aggressor's relationships would all load strongly. For the assertive factor, we expected that stating that the provocation crossed limits and seeking an explanation would load strongly. Previous work has indicated that youth see spending time near a teacher as a behavior associated with withdrawal (e.g., Younger et al. 2000). As such, we expected that telling a teacher would load on our hypothesized avoidance factor.

Three strategies had two plausible factor loadings. As described earlier, ending one's relationship with the aggressor might be aggressive, avoidant, or both. Responses involving saying or doing nothing are often classified as avoidant (e.g., Rudolph et al. 1994); however, they may also contain an element of assertiveness, in that youth may be choosing to withdraw, even though they believe they could enact a more confrontational response, because they expect doing nothing will result in the best outcome. As such, doing nothing may also load on the assertiveness factor. Finally, the strategy combining seeking an explanation and verbal aggression could load on either or both of the aggression and assertiveness factors.

We assessed the convergent validity of the PPI-MC by examining the extent to which the latent factors of aggression, avoidance, and assertion were associated with children's report of their aggressive behavior and their anger regulation, as indexed by their report of their dysregulated anger expression and their ability to cope with anger. It was expected that children's self-reported aggression would correlate positively with endorsement of aggressive responses to provocation. A significant amount of work has linked children's ability to manage their feelings of anger to aggressive behavior problems (e.g., Graham et al. 1992; Zeman et al. 2002). As such, we expected that ability to cope with anger would be associated negatively with aggressive behavior, whereas dysregulated anger expression would be associated positively with aggressive behavior. Furthermore, recent work has shown that feeling angry is associated negatively with children's reports that they would let a conflict go or try to work it out (MacEvoy and Asher, in press), suggesting that ability to cope with anger may be positively associated with avoidance and assertion behaviors, whereas dysregulated anger will be negatively associated with these responses.

\section{Methods}

Participants

Original Sample Participants were 392 youth in grades 6 to 8 attending a middle school in the northeastern United States $(M$ age $=12.69$, range $=10.83-15.00, S D=0.95)$. The average family income of students in this school was $\$ 18,404$, and $62 \%$ of students received free or reduced-cost lunch. Parents of all students enrolled at the school $(N=$ 884) were asked to provide written consent for their children to participate in the study. Parents who did not return consent forms were contacted by telephone to obtain consent. One hundred and thirty five parents $(15 \%)$ did not 
provide consent. Of the remaining students, 392 (44\%) completed the provocation measure. Demographic characteristics of the final sample are presented in Table 1.

Cross-validation Sample Measures were presented to children in the same school 7 months later, allowing us to cross-validate our model. Consent was obtained for 749 of 884 youth (85\%), and 327 completed the PPI-MC (37\%). Of these, 124 children completed the measure only at the second assessment. To ensure independence of the original and cross-validation samples, only these 124 children were included in the analysis. Demographic characteristics of the cross-validation sample were as follows: $M$ age $=13.11$ years (range $=10.83-15.58$ years, $S D=1.11$ ); $45 \%$ male; $44 \%$ in grade $6,26 \%$ in grade 7 , and $30 \%$ in grade $8 ; 12 \%$ nonHispanic white, $12 \%$ African American, 60\% Hispanic, and $7 \%$ other ethnicity.

\section{Measures}

The Peer Provocation Inventory - Multiple-Choice (PPI$M C)$ includes 11 provocation scenarios which depict being shoved, being ignored, having property damaged, being excluded from a party, being called a name, having a rumor spread about you, being teased about appearance, being teased about doing well in school, having a secret told about you, being threatened physically, and being teased about doing poorly in school. It is important to include a number of different scenarios, because previous research has demonstrated that youth responses vary as a function of provocation type (Dirks et al. 2007b); thus, including only one type of situation, or asking youth to report how they would respond to provocation, in general, will not provide enough information about behavior. Rather, it would be more edifying to examine how often they use a particular behavior across a number of scenarios, as youth who struggle in a greater number of situations are more likely to experience significant interpersonal difficulties (see McFall 1982). Each situation was chosen because (a) it was identified specifically in a study by Farrell et al. (1998) as relevant for lower-income youth; or (b) it often appears in other measures of social information processing (e.g., Crick 1995). Youth in a previous study identified these 11 situations as frequently occurring, difficult to manage, and critical (Dirks 2007). The relevance of the situations for the current sample was assessed by asking children to rate how often they had experienced each of the situations in the past year at school on a scale from 0 (never) to 4 (more than 10 times).

Each scenario describes the aggressor as a "good friend." Previous work has demonstrated that children's responses
Table 1 Descriptive statistics for measured variables
RPEQ Revised Peer Experiences Questionnaire; CAMS Children's Anger Management Scale.

${ }^{a}$ Number of situations in which a given type of response was selected.

\begin{tabular}{|c|c|c|c|c|}
\hline Variable & $M$ & $S D$ & $\mathrm{n}$ & $\%$ of sample \\
\hline Male & - & - & 190 & 49 \\
\hline Grade 6 & - & - & 106 & 27 \\
\hline Grade 7 & - & - & 139 & 36 \\
\hline Grade 8 & - & - & 147 & 38 \\
\hline Non-Hispanic White & - & - & 64 & 16 \\
\hline Hispanic & - & - & 206 & 53 \\
\hline African-American & - & - & 42 & 11 \\
\hline Other Ethnicity & - & - & 80 & 20 \\
\hline RPEQ & 5.53 & 6.00 & - & - \\
\hline CAMS-Emotion Regulation Coping & 8.09 & 2.02 & - & - \\
\hline CAMS—Dysregulated Expression & 5.51 & 1.67 & - & - \\
\hline \multicolumn{5}{|l|}{ Response to peer provocation ${ }^{\mathrm{a}}$} \\
\hline Physical aggression & 3.70 & 3.41 & - & - \\
\hline Verbal aggression & 3.16 & 2.71 & - & - \\
\hline Ending relationship with aggressor & 1.76 & 2.42 & - & - \\
\hline Damaging aggressor's relationship with others & 2.87 & 2.02 & - & - \\
\hline Doing nothing & 1.42 & 2.19 & - & - \\
\hline Seeking explanation & 2.76 & 3.01 & - & - \\
\hline Telling adult & 1.13 & 2.11 & - & - \\
\hline Stating provocation crossed limits & 3.51 & 2.99 & - & - \\
\hline Seeking explanation + verbal aggression & 2.76 & 2.82 & - & - \\
\hline \multicolumn{5}{|l|}{ Other } \\
\hline Completed questionnaire first & - & - & 213 & 54 \\
\hline
\end{tabular}


change as a function of aggressor identity (e.g., Burgess et al. 2006), necessitating that the provocateur be identified. We chose to focus on conflict within friendships in light of the importance of maintaining these relationships for children's well-being (e.g., Rose and Asher 1999). As described previously, each vignette was paired with nine possible behavioral responses: One strategy from each of the eight descriptive coding categories and one response combining verbal aggression and seeking an explanation. In our earlier work, several types of strategies were not generated in response to all situations. For example, physically aggressive responses only occurred in response to physically aggressive provocation. In these cases, we created responses based on the situation. Although the same nine categories were represented, the specific strategies for each scenario were not standardized to describe exactly the same behavior. Creating vignette-specific responses was designed to enhance the external validity of the measurement approach by increasing the realism of the strategies. Gender of the characters in the stories was matched to the participant.

The aggressor version of the Revised Peer Experiences Questionnaire (RPEQ; Prinstein et al. 2001) was used to operationalize aggression. Although originally used with adolescents, this measure has been given to children as young as 11 years (e.g., Dempsey et al. 2009). This measure consists of 18 items asking children to rate how often they have engaged in behavior during the last year (e.g., "I threatened to hurt or beat up another kid") on a 5-point Likert scale ( $1=$ never, to $5=a$ few times a week). For this study, the items on the three aggression subscales - Overt Aggression, Reputational Aggression, and Relational Aggression-were combined to form a total aggression score. The psychometric soundness of the RPEQ has been demonstrated (Prinstein et al. 2001), and in the current study internal-consistency reliability of this scale was excellent, $\alpha=.91$.

The Children's Anger Management Scale (CAMS, Zeman et al. 2002) was used to measure anger regulation. Children rate 11 items on a 3 -point scale ( $1=$ hardly ever to $3=$ often $)$, and these items are summed to form three scales. We were interested in the Emotion Regulation Coping scale, which assesses children's self-perceived ability to cope with their anger constructively, and the Dysregulated Expression scale, which measures children's engagement in culturally inappropriate displays of anger. The reliability and validity of these scales have been demonstrated (Zeman et al. 2002). In the current investigation, Cronbach's $\alpha$ s were .71 and .59 for the Emotion Regulation Coping and Dysregulated Expression scales, respectively.

\section{Procedure}

Measures were administered as part of a battery designed to evaluate a social-emotional learning curriculum. All proce- dures were approved by the Institutional Review Board. Data were collected from participants in a control school that did not receive the intervention. On the multiple-choice inventory, participants were instructed to read the vignette and then circle each strategy they would actually use. This approach was adopted because our previous work indicated that many youth incorporated multiple strategies in their responses (Dirks et al. 2007a). Participants completed a second measure asking them to evaluate the efficacy of different responses to provocation, results of which are not presented here. Order of presentation of the two measures was counterbalanced. These two measures were not a focus of the primary study and thus were included last in the battery. This placement likely contributed to the completion rates, as fatigue and time constraints may lead a number of participants to "drop out" while completing a measurement battery. Rates of completion were similar for questionnaires administered immediately prior to the provocation measures.

\section{Data Analysis}

Given the percentage of participants who did not complete the measure, chi-square analyses were conducted to assess if completion was associated with gender, grade, and ethnicity. Next, to assess the relevance of the situations, we calculated the number of participants who reported experiencing the situation at least once in the last year. Our central research questions concerned the latent structure underlying youth endorsement of responses to peer provocation. We began by screening youth responses on the PPI-MC. A small number of participants endorsed large numbers of responses that likely did not reflect strategies they would actually use (e.g., circling eight of nine responses), and these cases (5\% in the original sample, $3 \%$ in the cross-validation sample) were removed. Descriptive statistics indicated that several variables had significantly non-normal distributions; for this reason we used bootstrapping techniques to calculate the standard errors in all analyses.

Factor-analytic techniques were used to analyze the strategies youth endorsed across the 11 situations. The measurement model included nine indicator variables, each representing the total number of situations in which participants endorsed a given strategy. For example, participants selecting physically aggressive responses in four situations received a score of four on the physical aggression variable. Analyses were conducted using a specification-search procedure in AMOS 18.0 (Arbuckle 2009), which is undertaken to identify paths that exist in the true population model but are not specified in the original hypothesized model (Schumacker 2006). Based on our hypotheses, we created three latent factors: aggression, 
avoidance, and assertion. We then specified a model in which we set required factor loadings for each response we expected would load on only one factor: specifically, we allowed physical aggression, verbal aggression, and damaging the aggressor's relationship with others to load freely on the aggression factor, telling an adult to load freely on the avoidance factor, and seeking an explanation and stating that the provocation crossed limits to load freely on the assertion factor. We then let every other possible factor loading be optional. The specification-search procedure fits every possible subset of the optional paths (Arbuckle 2009), and the resulting models are compared using a number of fit indices. This approach allows us to capitalize on the advantages of a confirmatory approach, such as determining the statistical significance of the factor loadings (Brown 2006), but also allows us to assess all possible loadings, as would occur in an exploratory factor analysis. Following this analysis, we examined the generalizability of the model by fitting it in an independent sample of children. Finally, to assess the convergent validity of the PPI-MC, we examined the zero-order correlations between the three latent factors and self-reported aggressive behavior and anger regulation.

\section{Results}

Representativeness of Samples

Chi-square analyses revealed that in the original sample ethnicity was associated significantly with completion, $\chi 2(3)=23.07, p<.01$. In the cross-validation sample, completion was associated with ethnicity, $\chi 2(3)=12.15, p<.01$, and gender, $\chi 2(1)=6.53, p<.05$. In both samples, it appeared that non-Hispanic white participants $(79 \%, 60 \%)$ were more likely to complete the measures than AfricanAmerican participants $(66 \%, 52 \%)$ and Hispanic participants $(55 \%, 40 \%)$. In the cross-validation sample, girls $(50 \%)$ were more likely to complete the measure than boys $(40 \%)$. The potential implications of these differences are examined in the discussion section.

\section{Representativeness of Situations}

The percentage of participants who reported experiencing the situation at least once in the last year at school was as follows: being shoved, 56.6\%; being ignored, 57.4\%; having property damaged, $34.8 \%$; being excluded from a party, $32.3 \%$; being called a name, $50.9 \%$; having a rumor spread about you, 53.7\%; being teased about appearance, $58.0 \%$; being teased about doing well in school, 38.9\%; having a secret told about you, 51.7\%; being threatened physically, 46.3\%; being teased about doing poorly in school, $35.8 \%$.

\section{Examination of Hypothesized Latent Structure}

Zero-order correlations among all measured variables are presented in Table 2. Results of the specification search indicated that there were three equivalent optimal models, based on Akaike's Information Criterion (0.00) and the BrowneCudeck Criterion (0.00; Bayes Information Criterion $=2.00$; $\chi 2(18)=1.30, p=.18)$. Review of these models revealed that they differed in their treatment of the loadings of three strategies on the aggression factor: seeking an explanation, stating that the provocation crossed limits, and seeking an explanation + verbal aggression. These models were identical in all other regards. When deciding between equivalent models, Schumacker (2006) noted that it is important to be guided by theoretical considerations. As described previously, we expected that seeking an explanation + verbal aggression might load on the aggression factor. In addition, the close relationship between assertive and aggressive behavior (see Ostrov et al. 2006) makes a positive relationship between stating the provocation crossed limits and the aggression factor plausible. The final model is presented in Fig. 1; it was selected over models that retained a negative loading of seeking an explanation on the aggression factor. The fit of the selected model was adequate (see Hu and Bentler 1999): $\mathrm{NFI}=.99, \mathrm{RFI}=.98, \mathrm{IFI}=1.00, \mathrm{TLI}=.99, \mathrm{CFI}=1.00$, and RMSEA $=.028(90 \% \mathrm{CI}=.000-.058)$. The Bollen-Stine bootstrap statistic is a modified version of the $\chi^{2}$ goodness-of-fit statistic (Byrne 2001). It tests the null hypothesis that the model is correct, and in this case was not significant, $p=.39$, suggesting adequate model fit.

\section{Cross-Validation of Measurement Model}

The multiple-choice measure was re-administered in the same school 7 months after the first data collection. Based on inspection of the previous data, minor modifications were made to the measure. Most notably, we found unexpectedly high endorsement of physical aggression in responses to situations in which youth in a previous study had generated no physically aggressive responses. This may have been due to youth finding the responses comical (e.g., "shove him out of his desk"). These responses were revised to be less humorous (e.g., "get in her face"). Data collection procedures were identical. We fit the final measurement model depicted in Fig. 1, substituting indicator variables collected at the second time point. Model fit was again adequate: Bollen-Stine bootstrap $p=.48, \mathrm{NFI}=.95, \mathrm{RFI}=.89, \mathrm{IFI}=.99, \mathrm{TLI}=.98$, $\mathrm{CFI}=.99$, and $\mathrm{RMSEA}=.039(90 \% \mathrm{CI}=.000-.096)$. 


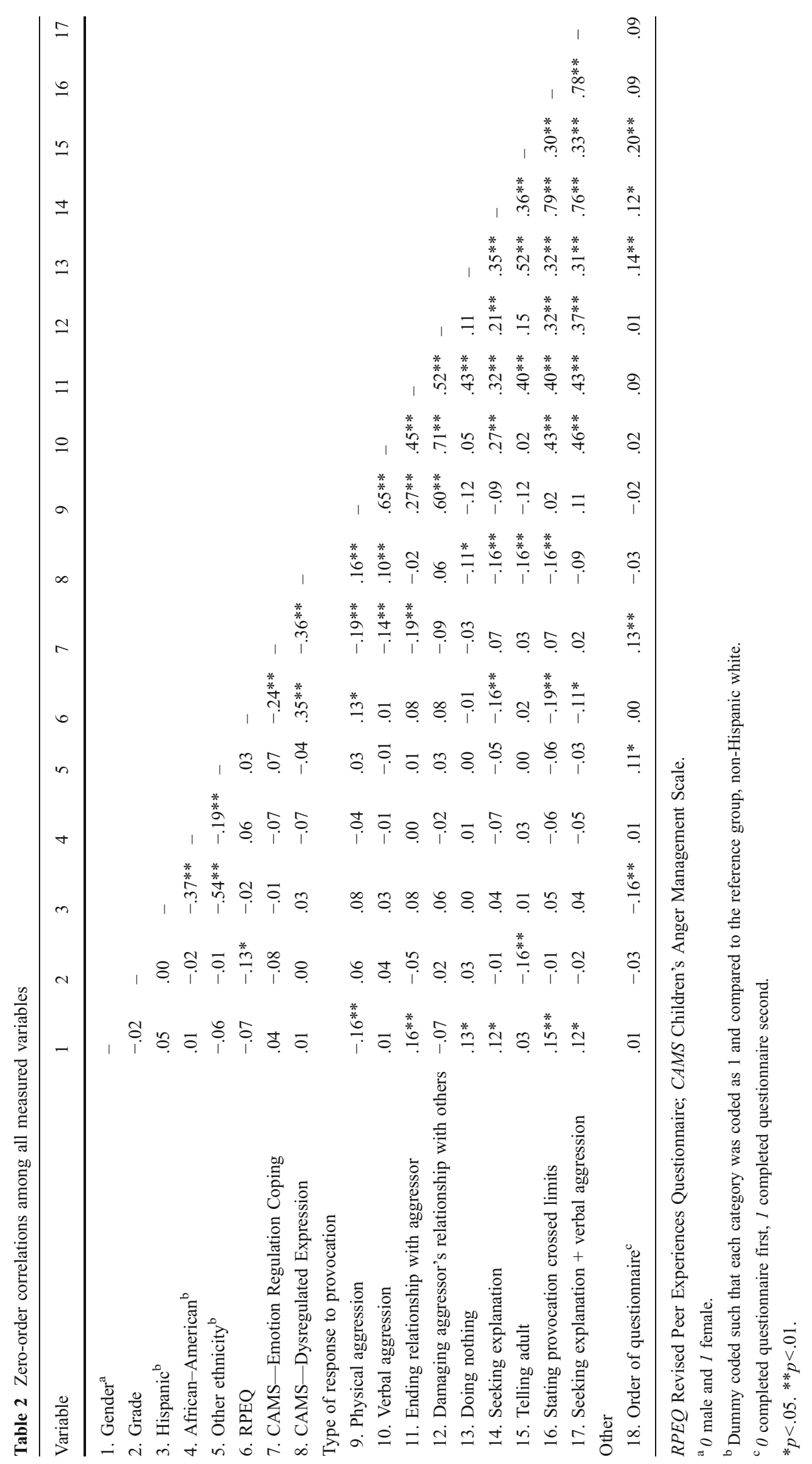


Fig. 1 Measurement model fit on original sample, including standardized factor loadings among behavioral strategies and the latent factors of aggression, avoidance, and assertion

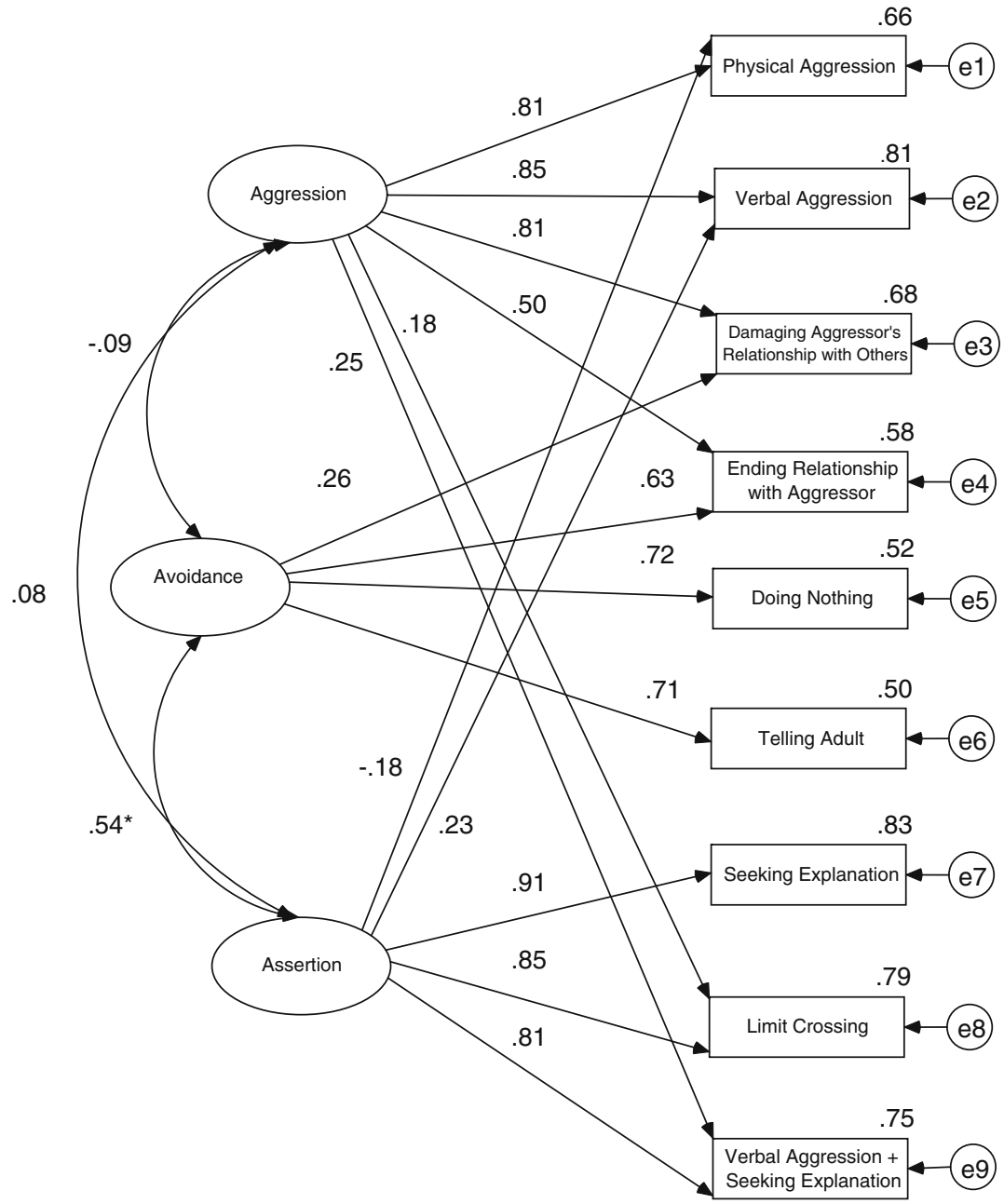

Note. Ninety-five percent bias-corrected bootstrap confidence intervals were obtained for all standardized factor loadings. All standardized coefficients were significant, $p<.05$.

$* p<.05$

Associations among Aggression, Anger, and Youth Endorsement of Aggressive, Avoidant, and Assertive Responses

To examine the convergent validity of the measure, we constructed a second model in which we assessed the zeroorder correlations between self-reported aggressive behavior (as indexed by the RPEQ), anger coping, and dysregulated anger expression (measured with the CAMS), and each of the three latent factors. With alpha set at .006 (.05/9), a significant negative association existed between the aggression factor and coping with anger, $r=-.20$, and a significant positive association existed between the aggression factor and dysfunctional display of anger, $r=.16$. Both the assertion and avoidance factors were associated negatively with dysfunctional display of anger, $r s=-.19$. Self-reported aggressive behavior was also associated negatively with the assertion factor, $r=-.20$.
Unexpectedly, self-reported aggressive behavior was not associated with the endorsement of aggressive responses on the PPI-MC. We posited that this null relationship might be due to the rating scale on the RPEQ. The RPEQ asks children to rate specifically how often they engage in aggressive behaviors; thus, the association between children's ratings on this measure and their endorsement of responses to peer provocation on the PPI-MC may depend, in part, on how often they experience the provocation situations. Children who would use aggression if provoked but never experience provocation would not be engaging in aggressive behavior; in these cases, the correlation between self-reported aggressive behavior and endorsement of aggressive responses to provocation would be low. To examine this possibility, we constructed new variables for each response type loading primarily on the aggression factor by multiplying whether or not youth selected a given response in each situation by the number of times they reported experiencing that situation, and 
then summing across situations. Self-reported aggression was positively associated with each of these variables: physical aggression, $r=.30, p<.01$; verbal aggression $r=.17, p<.01$; and damaging aggressor's relationship with others, $r=.20$, $p<.01$. These results suggest that endorsement of aggressive responses on the PPI-MC is associated with self-reported aggressive behavior on the RPEQ.

\section{Discussion}

This study used a multiple-choice measure to examine the latent structure of young adolescents' responses to peerprovocation scenarios that occur in their everyday lives. Using factor-analytic techniques, we found that a model consisting of three latent constructs - aggression, avoidance, and assertion-characterized adequately the structure of youth responses. This model was cross-validated in a second sample. This analysis provides further evidence for the validity of this commonly employed tri-partite categorization of youth behavior. Previous investigators have classified children's behaviors into these categories a priori (e.g., Quiggle et al. 1992); however, no work has examined whether children's responses coded at a more micro, descriptive, level, actually conform to this structure. Our analysis provides empirical support for the adequacy of these three broadband categories in capturing the range of strategies children employ when confronted with provocation.

In the final, three-factor model, eight of nine strategies showed loadings exceeding .70 on one of the three factors, with several strategies showing small loadings on a second factor. There is increasing recognition that simple structure (i.e., each indicator variable loading significantly on only one factor) may not be a realistic goal for many measures, as a number of small cross-loadings that are either theoretically understandable or result from the design of the measure are likely to emerge (see Asparouhov and Muthén 2009). Such cross-loadings were perhaps especially likely in the case of the PPI-MC, which was designed not to identify clear-cut examples of aggressive, avoidant, or assertive behavior, but to include a naturalistic representation of the behaviors youth use when they are provoked.

Indeed, at the outset, three strategies - ending relationship with the aggressor, doing nothing, and seeking an explanation + verbal aggression-appeared to combine elements of two factors. Some researchers have included ending one's relationship with the aggressor as a type of relational aggression (e.g., Delveaux and Daniels 2000). Our analysis suggested that this behavior loaded significantly on both the aggression and avoidance factors, with the magnitude of these loadings being similar, calling into question the categorization of this behavior as purely aggressive. The other strategy typically viewed as relationally aggressive, damaging the aggressor's relationships with others, also had a small, but significant, loading on the avoidance factor, perhaps reflecting the fact that unlike physical and verbal aggression, this strategy does not necessarily involve a direct confrontation. "Doing nothing" correlated most strongly with ending one's relationship with the aggressor and telling the teacher, a pattern more consistent with the interpretation of doing nothing as avoidance, rather than as an assertive behavior.

The combination of seeking an explanation and verbal aggression loaded strongly on the assertion factor, with a small, but significant, loading on the aggression factor, suggesting that the young adolescents in our sample may be employing these responses as a minor component of an aggressive style. In general, the factor loadings in the final model were consistent with previous theorizing concerning the fuzzy boundaries between assertion and aggression (see Ostrov et al. 2006). Stating that the provocation crossed limits loaded primarily on the assertion factor, but also had a small positive loading on the aggression factor; similarly, verbal aggression loaded primarily on the aggression factor but had a small positive loading on the assertion factor.

It may increase the utility of the PPI-MC to incorporate these cross-loadings explicitly when scoring the measure. One approach to totaling the PPI-MC would be to calculate aggression, avoidance, and assertion scores using responses that had primary loadings on each of the factors (e.g., children's total assertion scores would be the number of times they endorsed seeking an explanation, stating that the provocation crossed limits, and seeking an explanation + verbal aggression). Alternatively, strategies with loadings on a second factor could be counted towards two totals (e.g., endorsing seeking an explanation + verbal aggression in a particular situation would add one to both the assertion and aggression scores). The first strategy treats crossloadings as non-existent, and the second treats them as equivalent to primary loadings. A more accurate picture may emerge from calculating weighted sums for each factor by multiplying a child's total endorsement of each response type (i.e., the number of situations in which they endorsed the strategy) by the factor weights, which are obtained through regression of the latent factors on the indicator variables (Arbuckle 2009; weights available from the first author). This approach to scoring would better capture the complexities present in children's social behaviors.

We obtained preliminary evidence for the convergent validity of the PPI-MC by examining the associations among the responses youth endorsed and their self-reported aggressive behavior and anger regulation. Aggressive behavior was negatively associated with the assertion factor, but unexpectedly was not associated with the aggression factor. We conducted secondary analyses in 
which we examined the association between aggressive behavior and aggressive responses to provocation, taking into account how often youth reported experiencing each provocation situation. These variables provided a tighter fit conceptually to the items on the RPEQ, and indeed, the relationships were then significantly positive. As expected, aggressive responses on the PPI-MC were associated negatively with anger-coping skills and positively with dysregulated anger expression; in contrast, selection of both assertive and avoidant strategies was associated negatively with dysregulated anger expression. The size of these effects was consistent with those seen in other studies using vignette-based measures of youth behavior (e.g., Dodge et al. 2002; Hughes et al. 2004; Quiggle et al. 1992; Wichmann et al. 2004). Other studies have found larger associations; however, methodological differences may account, at least in part, for these discrepancies. For example, Deluty (1979) correlated self-, peer-, and teacher-reports of children's behavior in specific interpersonal situations. Use of similar items across informants likely resulted in larger associations. Chung and Asher (1996) compared extreme groups (e.g., children receiving the most aggressive nominations from classmates versus those receiving the most prosocial nominations) on their endorsement of different behavioral strategies, an approach that may also have yielded greater effects.

Several limitations must be noted. A significant number of available participants did not complete the measure. Youth experiencing aggressive behavior difficulties may be more likely not to complete questionnaires (e.g., Metropolitan Area Child Study Research Group 2002). If this is the case, the associations among self-reported aggression, anger regulation, and responses to provocation may have been underestimated. As such, our estimates would reflect a conservative, rather than a liberal, bias. In addition, our analyses suggested that the three-factor model identified in the first sample also fit in a second sample, providing preliminary evidence of generalizability. An additional limitation is our reliance on self-report data. Evidence from other studies has suggested that youth's responses to vignette-based measures correspond with other informants' ratings of behavior (e.g., Chung and Asher 1996; Deluty 1979). An important next step will be to corroborate youth responses with peer, parent, and teacher ratings. Test-retest reliability should also be established.

This study extended understanding of youth responses to peer provocation - a key class of social situations - by examining the latent structure of the strategies youth select when confronted with a range of peer aggression. This analysis provided empirical support for the classification of youth behaviors into the broadband categories of aggression, avoidance, and assertion; however, our findings also suggested that many of the strategies youth actually use to manage provocation are not "pure" examples of one of these categories. These responses are potentially of interest, however, both clinically and socially. As such, these results suggest that when developing task-based measures of youth social functioning, it will be important to sample the range of behaviors actually endorsed by youth in those interpersonal contexts. Ultimately, the results of this line of inquiry may inform the development of interventions designed to improve youth social functioning. Identifying precisely which situations prove troublesome for youth, as well as how they struggle will provide clear targets for intervention (Dirks et al. 2007a).

Acknowledgments During preparation of this article, the first author was supported by a post-doctoral fellowship from the Ontario Mental Health Foundation.

Open Access This article is distributed under the terms of the Creative Commons Attribution Noncommercial License which permits any noncommercial use, distribution, and reproduction in any medium, provided the original author(s) and source are credited.

\section{Appendix}

Sample Vignette and Responses from Multiple-Choice Measure

You are walking by yourself up to the door of the school before class in the morning. There are lots of students outside, and some of the teachers too. As you get to the door you see a good friend of yours. She is walking into the school. You say hi to her. She turns around, looks right at you, and says "why are you talking to me?" then turns away from you and walks quickly away.

What would you say or do if this happened to you?

Please fill in the circle next to everything that you would really say or do.

(1) Say "What's wrong with you?"

(2) Tell the teacher what $\mathrm{s} /$ he did.

(3) Tell all our friends what $\mathrm{s} /$ he did and get them on my side.

(4) Never speak to him/her again.

(5) Say "What did you do that for?

(6) Call him/her a rude name.

(7) Say "You should apologize."

(8) Shove him/her back.

(9) Walk away

\section{References}

Arbuckle, J. L. (2009). AMOS 18 user's guide. Chicago: Amos Development Corporation.

Asparouhov, T., \& Muthén, B. (2009). Exploratory structural equation modeling. Structural Equation Modeling, 16, 397-438. 
Brown, T. A. (2006). Confirmatory factor analysis for applied research. New York: Guilford.

Burgess, K. B., Wojslawowicz, J. C., Rubin, K. H., Rose-Krasnor, L., \& Booth-LaForce, C. (2006). Social information processing and coping strategies of shy/withdrawn and aggressive children: does friendship matter? Child Development, 77, 371-383.

Byrne, B. M. (2001). Structural equation modeling with AMOS: Basic concepts, applications, and programming. Mahwah: Lawrence Erlbaum.

Card, N. A., \& Hodges, E. V. E. (2008). Peer victimization among schoolchildren: correlations, causes, consequences, and considerations in assessment and intervention. School Psychology Quarterly, 23, 451-461.

Chung, T.-Y., \& Asher, S. R. (1996). Children's goals and strategies in peer conflict situations. Merrill-Palmer Quarterly, 42, 125-147.

Crick, N. R. (1995). Relational aggression: the role of intent attributions, feelings of distress, and provocation type. Development and Psychopathology, 7, 313-322.

Crick, N. R., \& Nelson, D. A. (2002). Relational and physical victimization within friendships: nobody told me there'd be friends like these. Journal of Abnormal Child Psychology, 30, 599-607.

Deluty, R. H. (1984). Behavioral validation of the children's action tendency scale. Journal of Behavioral Assessment, 6, 115-130.

Deluty, R. H. (1979). Children's action tendency scale: a self-report measure of aggressiveness, assertiveness, and submissiveness in children. Journal of Consulting and Clinical Psychology, 47, 1061-1071.

Delveaux, K. D., \& Daniels, T. (2000). Children's social cognitions: physically and relationally aggressive strategies and children's goals in peer conflict situations. Merrill-Palmer Quarterly, 46, 672-692.

Dempsey, A. G., Sulkowski, M. L., Nichols, R., \& Storch, E. A. (2009). Differences between peer victimization in cyber and physical settings and associated psychosocial adjustment in early adolescence. Psychology in the Schools, 46, 962-972.

Dirks, M. A. (2007). Situation- and judge-specific factors in the conceptualization and measurement of youth social functioning: The case of peer provocation. Unpublished doctoral dissertation.

Dirks, M. A., Treat, T. A., \& Weersing, V. R. (2007a). Integrating theoretical, measurement, and intervention models of youth social competence. Clinical Psychology Review, 27, 327-347.

Dirks, M. A., Treat, T. A., \& Weersing, V. R. (2007b). The situation specificity of youth responses to peer provocation. Journal of Clinical Child and Adolescent Psychology, 36, 621-628.

Dodge, K. A., Laird, R., Lochman, J. E., Zelli, A., \& the Conduct Problems Prevention Research Group. (2002). Multidimensional latent construct analysis of children's social information processing patterns: correlations with aggressive behavior problems. Psychological Assessment, 14, 60-73.

Elledge, L. C., Cavell, T. A., Ogle, N. T., Malcolm, K. T., Newgent, R. A., \& Faith, M. A. (2010). History of peer victimization and children's response to school bullying. School Psychology Quarterly, 25, 129-141.

Farrell, A. D., Ampy, L. A., \& Meyer, A. L. (1998). Identification and assessment of problematic interpersonal situations for urban adolescents. Journal of Clinical Child Psychology, 27, 293-305.

Goldfried, M. R., \& D'Zurilla, T. J. (1969). A behavioral analytic model for assessing competence. In C. D. Spielberger (Ed.), Current topics in clinical and community psychology: Vol. 1 (pp. 151-195). New York: Academic.

Graham, S., Hudley, C., \& Williams, E. (1992). Attributional and emotional determinants of aggression among African-American and Latino young adolescents. Developmental Psychology, 28, 731-740.
Hu, L., \& Bentler, P. M. (1999). Cutoff criteria for fit indices in covariance structure analysis: conventional criteria versus new alternatives. Structural Equation Modeling, 6, 1-55.

Hughes, J. N., Meehan, B. T., \& Cavell, T. A. (2004). Development and validation of a gender-balanced measure of aggressionrelevant social cognition. Journal of Clinical Child and Adolescent Psychology, 33, 292-302.

Kochenderfer, B. J., \& Ladd, G. W. (1997). Victimized children's responses to peers' aggression: behaviors associated with reduced versus continued victimization. Development and Psychopathology, 9, 59-73.

Kochenderfer-Ladd, B. J. (2004). Peer victimization: the role of emotions in adaptive and maladaptive coping. Social Development, 13, 329-349.

MacEvoy, J. P., \& Asher, S. R. (in press). When friends disappoint: Boys' and girls' responses to transgressions of friendship expectations. Child Development.

McFall, R. M. (1982). A review and reformulation of the concept of social skills. Behavioral Assessment, 4, 1-33.

Metropolitan Area Child Study Research Group. (2002). A cognitiveecological approach to preventing aggression in urban settings: initial outcomes for high-risk children. Journal of Consulting and Clinical Psychology, 70, 179-194.

Ollendick, T. H. (1983). Development and validation of the children's assertiveness inventory. Child and Family Behavior Therapy, 5, $1-15$.

Ostrov, J. M., Pilat, M. M., \& Crick, N. R. (2006). Assertion strategies and aggression during early childhood: a short-term longitudinal study. Early Childhood Research Quarterly, 21, 403-416.

Patterson, G. R., Littman, R. A., \& Bricker, W. (1967). Assertive behavior in children: a step toward a theory of aggression. Monographs of the Society for Research in Child Development, 32(5), 1-43.

Prinstein, M. J., Boergers, J., \& Vernberg, E. M. (2001). Overt and relational aggression in adolescents: social-psychological adjustment of aggressors and victims. Journal of Clinical Child Psychology, 30, 479-491.

Quiggle, N. L., Garber, J., Panak, W. F., \& Dodge, K. A. (1992). Social information processing in aggressive and depressed children. Child Development, 63, 1305-1320.

Rose, A. J., \& Asher, S. R. (1999). Children's goals and strategies in response to conflicts within a friendship. Developmental Psychology, 35, 69-79.

Rudolph, K. D., Hammen, C., \& Burge, D. (1994). Interpersonal functioning and depressive symptoms in childhood: addressing the issues of specificity and comorbidity. Journal of Abnormal Child Psychology, 22, 355-371.

Schumacker, R. E. (2006). Conducting specification searches with AMOS. Structural Equation Modeling, 13, 118-129.

Tapper, K., \& Boulton, M. J. (2004). Sex differences in levels of physical, verbal, and indirect aggression amongst primary school children and their associations with beliefs about aggression. Aggressive Behavior, 30, 123-145.

Troop-Gordon, W., \& Asher, S. R. (2005). Modifications in children's goals when encountering obstacles to conflict resolution. Child Development, 76, 568-582.

Wichmann, C., Coplan, R. J., \& Daniels, T. (2004). The social cognitions of socially withdrawn children. Social Development, 13, 377-392.

Younger, A. J., Schneider, B. H., Wadeson, R., Guirguis, M., \& Bergeron, N. (2000). A behaviour-based peer-nomination measure of social withdrawal in children. Social Development, 9, 544-564.

Zeman, J., Shipman, K., \& Suveg, C. (2002). Anger and sadness regulation: predictors to internalizing and externalizing symptoms in children. Journal of Clinical Child and Adolescent Psychology, 31, 393-398. 